\title{
Scanning Electron Microscopy study of the standard sterilization procedure applied to dental instruments
}

\author{
M. L. Redígolo, ${ }^{*}$ P. G. R. Cardoso, ${ }^{*}$ M. B. S. Posso, ${ }^{*}$ and S. Koury ${ }^{*}$ \\ *IP\&D, Univap, Av. Shishima Hifumi, 2911, São José dos Campos, SP, Brazil, 12244-000
}

The potential for contamination of rotary dental instruments has become a topic of considerable discussion and continuing investigation. The possibility of retracting fluids into the instrument and the retention of organisms on or in the dental handpieces are a fact [1].

This work analyzes the sterilization conditions of ultra-high-speed air turbines through scanning electron microscopy (SEM) observing the internal parts of the dental handpiece ducts searching for points of corrosion and biofilms. The electron microscopy was performed with a JSM-5900 LV microscope (JEOL) under $15 \mathrm{kV}$ and $25 \mathrm{kV}$, at the Brazilian Synchrotron Light Laboratory. The samples were also submitted to Energy Dispersive Spectroscopy (EDS) to check for specific proteins characteristic of bacteria activity.

Ultra-high-speed air turbines rely on the principle of a rotor supported by miniature ball bearings. Only a perfect efficiency of all their parts allows these instruments to attain high speeds. Usually the rings and spheres inside the handpiece are made of a high carbon-chromium steel to satisfy these mechanical properties. However, the amount of chromium is not enough to ensure the presence of a highly protective chromium-oxide layer avoiding corrosion. Normally a thin iron-oxide film is present on the surface of these steels. The sterilizing procedures may be a cause of the decay of these turbines once the products employed can react with the iron oxide removing it and causing rapid corrosion of the carbon-chromium material [2].

For this study, three dental handpieces were analyzed. The first one was submitted to 1000 sterilizing cycles during its 5 year period of use. The second one was submitted to only 30 cycles of sterilization process. A brand new handpiece that was not submitted to any use or sterilization procedure represents the control sample. The standard sterilizing procedures here adopted include a cleaning process in enzyme detergents using an ultrasonic device with distilled water. Then the handpieces were submitted to a lubrication process with an aqueous solution (Premix (B) and to standard autoclaving $\left(121^{\circ} \mathrm{C}\right.$ during $\left.30 \mathrm{~min}\right)$ in steam sterilizers. Through the microscopy and a bacterial culture analysis, no biofilm was detected. While these procedures are effective in preventing the formation of the biofilm they are also considerably aggressive contributing to the handpieces corrosion process. Figure 1 shows the EDS results for debris found inside the handpiece air duct presenting traces of Chromium and other elements. The most used handpiece analyzed in this experiment presented a deteriorated internal surface for the water duct due to the corrosion process (figure $2 b$ ) differently from the aspect observed for the control handpiece (figure $2 a$ ).

\section{References}

[1] D. L. Leonard and D. G. Charlton, JADA 130 (1999) 1301

[2] E. Angelini, Quintessence International 23 (1992) 215. 


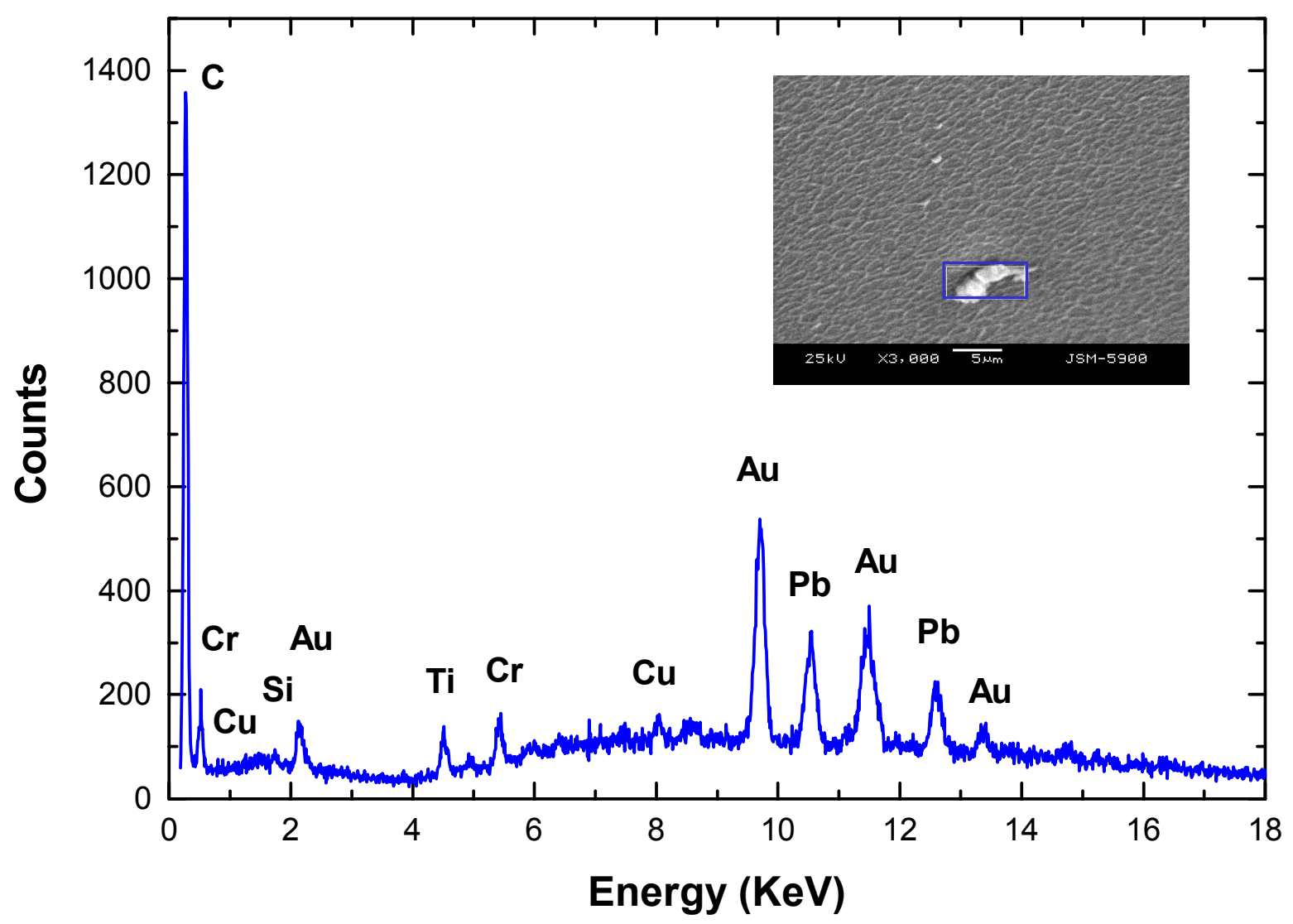

FIG. 1. EDS analysis of the marked region presented in the SEM photomicrograph (x 3000; $5 \mu \mathrm{m}$ bar)

a)
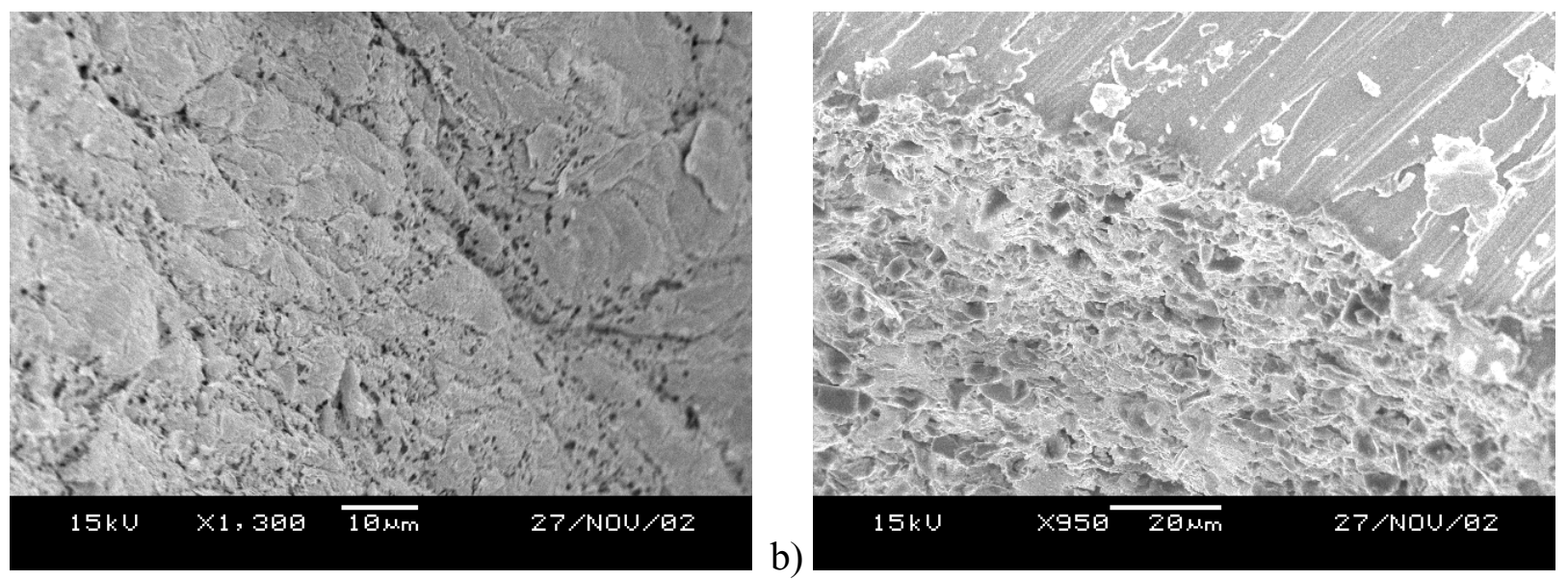

FIG. 2. SEM photomicrograph of the inner surface of the water duct for (a) the control sample (x 1300, $10 \mu \mathrm{m}$ bar) and for (b) the most used handpiece (x 90; $200 \mu \mathrm{m}$ bar). 